\title{
EFFECT OF ION-INDUCED ELECTRONS EMISSION ON THE PLASMA SHEATH VOLTAGE
}

\author{
R. Khanal \\ Central Department of Physics, Tribhuvan University, Kirtipur, Kathmandu, Nepal
}

\begin{abstract}
The effect of ion-induced electrons emission on the plasma sheath voltage for different values of ion induced electrons emission coefficient has been studied. Using a Kinetic Trajectory Simulation model of plasma sheaths, we calculate the particle densities reaching the wall and for given ion induced electrons emission coefficient the additional electrons emitted from the wall is obtained. Finally, the plasma sheath voltage (wall potential) is obtained by solving the Poisson equation. It has been observed that the potential decreases as the ion-induced electron emission coefficient increases as expected theoretically.
\end{abstract}

Key words: Plasma; Quasineutrality; Debye shielding, Sheath; Presheath; Bohm criterion.

\section{INTRODUCTION}

In all practical plasma devices, plasma interacts with the vessel (material walls). The interaction of plasma with the wall has been a matter of interest in all applications of plasma including the schemes to hamess the fusion energy.

Plasma contains ions as well as electrons but the mobility of the electrons is much greater and hence can reach the wall earlier than the ions. This makes the wall negatively charged Due to this, futher electrons going towards the wall are repelled whereas the ions are accelerated towards the wall. Thus near the wall, the electron density decreases much faster than that of ions and a positive space charge region is formed shielding the potential at the wall. This shielding is of the order of some electron Debye length. This space charge region of positive ions which is very short, compared to the characteristic length of plasma, is called the "Sheath". The sheath structure formed adjacent to a wall facing plasma contributes to the stability of the core plasma. Becanse of the sheath formed the negative potential at the wall can not penetrate deep into the core plasma and the core is virtually undisturbed.

For a sheath to exist, the in-streaming ions at the sheath entrance have to satisfy a condition called the "Bohm criterion" [1] [2] [3]. In it's kinetic form, this criterion reads,

$\left\{\frac{1}{v^{2}}\right\} \leq \frac{m^{r}}{k\left(\gamma^{\top} T_{w}^{i}+y^{d} T_{w}^{d}\right)}$

where $k$ is the Boltzmam constant, $\gamma^{i}$ and $\gamma^{2}$ are the ion and electron polytropic constants, respectively, and $T_{p s}^{f}$ and $T_{p s}^{\varepsilon}$ are the ion and electron temperatures at the presheath side of sheath edge, respectively. The condition (1) ensures the potential profile to be non-0scillatory at the sheath edge. In realistic cases, it is usually satisfied marginally, i.e., with the equality sign.

The Bohm criterion implies that we need an electric field in the region preceding the sheath in order to accelerate the ions. The region in the plasma where the acceleration of the ion begins is called the presheath. Thus the plasma confined in a vessel can generally be classified into three regions: the sheath, the presheath and the bulk plasma (cf. Fig. 1) [4]

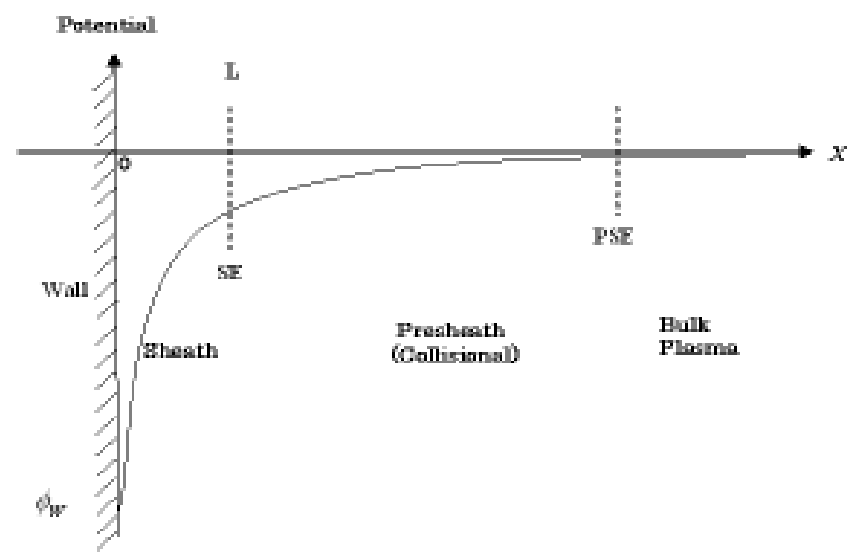

Fig. 1: General structure of the plasma wall transition region. The sheath and collisional presheath edges are denoted by SE and PSE, respectively

It is important to note that the sheath structure is also responsible for determining the energy and particle flow towards the wall and overall bulk plasma behavior. In the sheath region the plasma is significantly non-neutral although it is practically quasineutral at the sheath entrance and in the presheath region as well. As the wall is negatively charged even the Debye shielding camot counteract the potential due to the wall and a residue potential of the order $k T_{e} / m$ can always 
leak ont from the sheath which is the primary cause of ion acceleration towards the sheath from presheath region. Hence, a very small potential difference occurs in the presheath region, which is very large in terms of length compared to the sheath. This helps in sheath formation provided the ion velocity satisfies the Bohm criterion.

Recently, Schupfer et al have studied the effect of particleinduced electron emission on the plasma sheath voltage [5] In order to investigate this effect, a new model involving a collisionless kinetic sheath consistently coupled to a fluid presheath was developed [6]

In the present work, we have studied the effect of ion-induced-electron emission to the plasma wall potential. Here we wanted to understand how the property of ions reaching the wall can change the sheath potential. For this, we have assumed that the ion induced electron beams to be cold. The effect has been studied for the case without a magnetic field or the field being perpendicular to the wall. Using the Kinetic Trajectory Simulation (KTS) model of plasma sheaths we calculate the particle densities reaching the wall and for given ion induced electrons emission coefficient the additional electrons emitted from the wall is obtained. Finally, the plasma sheath voltage (wall potential) is obtained by solving the Poisson equation. It has been observed that the potential decreases as the ion-induced electron emission coefficient increases, as expected theoretically.

The characteristic feature of the KTS method is that the distribution functions of the particle species involved are calcnlated directly by solving the related kinetic equations along the respective collisionless particle trajectory [6-9]. In order to obtain the distribution function at any point $[x, v]$ of the phase-space we trace the related trajectories to some phasespace where the distribution is given.

\section{THE PLASMA SHEATH MODEL}

In the present work, we restrict ourselves to time-independent, collisionless electrostatic problems, as is appropriate for the sheath regions. The IdIV model of the plasma sheath is shown schematically in Fig. 2. The notation $1 d l v$ indicates the fact that our model is one-dimensional both in configuration and in velocity space. The magnetic field $\mathrm{B}$ is nomal to the wall and assumed to be "infinitely" strong, so that the IdIV approximation is appropriate

The simulation region considered is bounded by two parallel planes located at $x=0$ and $x=L$, and the plasma is assumed to consist of electrons and one species of singly charged ions. The two boundaries are specified as follows. The right hand boundary $(x=L)$ is the "sheath entrance", separating the non-neutral, collisionless sheath region $(x<L)$ from the quasi-neutral, collisional presheath region $(x>L)$, where as the left-hand boundary $(x=0)$ represents an absorbing wall. The potential value at $x=L$ is always chosen equal to zero.

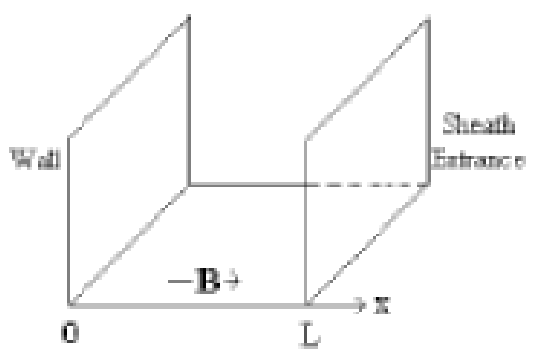

Fig. 2: Id'v plassma sheath model

We assume that the plasma particles (electrons and ions) enter the simulation region from the right-hand boundary with cut-off Maxwellian velocity distribution functions, that the left-hand boundary does not emit any particles, and that both boundaries are perfectly absorbing. Accordingly, the electron velocity distribution function is given by,

$f^{2}(x, v)=A^{e} \operatorname{exP}\left[-\left(\frac{v}{v_{j}^{e}}\right)^{2}+\frac{e \phi(x)}{k T_{f}^{v}}\right] \Theta\left[v_{e}^{e}(x)-v\right]$

where

$v_{c}^{e}(x)=\sqrt{\frac{2 e\left[\phi(x)-\phi_{0}\right]}{m^{e}}}$

is the electron cut-off velocity at $x$. The ion velocity distribution function at $x=L$ is given by;

$f^{i}(L, v)=A^{i} \exp \left[-\left(\frac{v-v_{\mathrm{raL}}^{\prime}}{v_{t r}^{\prime}}\right)^{2}\right] \Theta\left(v_{\mathrm{rL}}^{i}-v\right)$

where

$v_{\sigma^{\prime}}^{2}=\sqrt{\frac{2 k T_{f}^{2}}{m^{2}}}$,

is the species-s themal velocity, $v_{m L}^{i}$ is the ion "Maxwellianmaximum" velocity at $x=L$, and $v_{c L}^{f}$ (with $v_{c L}^{t}<0$ ) is the ion cut-off velocity at $x=L$. Here, $T_{j}^{e}$ and $T_{j}^{\prime}$ are the "formal" electron and ion temperatures.

Exact ion trajectories are followed, to calculate along them the ion distribution function, for the given ion injection distribution. The electrons, on the other hand, are assumed to have a half Maxwellian velocity distribution at injection, so that their density can be calculated analytically. Due to absorption at 
Table 1: Nomalized floating potential and comparison with theoretical valnes

\begin{tabular}{|c|c|c|c|c|}
\hline$\gamma^{e t}$ & $\begin{array}{l}n_{p s} \times 10^{18} \mathrm{~m}^{-3} \\
\text { (Presheath ion } \\
\text { density) }\end{array}$ & $\phi_{F}(V)$ & $\begin{array}{c}\widetilde{\phi}_{0} \\
\text { (Normalized potential, cf. Ref. } \\
{[5] \text { ) }}\end{array}$ & $\begin{array}{c}\widetilde{\phi}_{0} \\
\text { (Theoretical result } \\
[5])\end{array}$ \\
\hline 0.00 & 1.0000 & -29.2747 & $=2.7485$ & -2.9275 \\
\hline 0.02 & 1.0084 & -29.3476 & -2.7566 & -2.9348 \\
\hline 0.04 & 1.0168 & $=29.4199$ & $=2.7646$ & $=2.9420$ \\
\hline 0.06 & 1.0251 & -29.4909 & -2.7724 & -2.9491 \\
\hline 0.08 & 1.0335 & $=29.5621$ & $=2.7803$ & $=2.9562$ \\
\hline 0.10 & 1.0419 & -29.6328 & -2.7881 & -2.9633 \\
\hline 0.11 & 1.0461 & $=29.6679$ & $=2.7920$ & $=2.9668$ \\
\hline 0.12 & 1.0503 & -29.7029 & -2.7958 & -2.9703 \\
\hline 0.13 & 1.0545 & $=29.7378$ & $=2.7997$ & $=2.9738$ \\
\hline 0.14 & 1.0587 & $=29.7726$ & $=2.8035$ & -2.9773 \\
\hline 0.15 & 1.0629 & -29.8072 & -2.8073 & -2.9807 \\
\hline 0.20 & 1.0838 & $=29.9776$ & $=2.8261$ & $=2.9978$ \\
\hline 0.25 & 1.1048 & -30.1458 & $=2.8447$ & -3.0146 \\
\hline 0.30 & 1.1257 & -30.3102 & $=2.8628$ & -3.0310 \\
\hline 0.50 & 1.2096 & -30.9426 & -2.9323 & -3.0943 \\
\hline 0.80 & 1.3353 & -31.8170 & -3.0283 & -3.1817 \\
\hline
\end{tabular}

the wall, their full velocity distribution is a cut-off Maxwellian. Starting from an initial guess, the potential profile is iterated towards the final time-independent, self-consistent state [6].

\section{RESULTS AND DISCUSSION}

As the ions accelerated from the bulk plasma region strike the material wall, electrons are released and flow back into the plasma, giving rise to low plasma sheath voltage than without electron emission. The decrement on the plasma sheath voltage depends on coefficient of ion-induced electron emission. Fig. 3 shows the variation of potential for different values of ion-induced electron emission coefficient $\left(\gamma^{e t}\right)$. The potential decreases as the ion-induced electron emission coefficient increases. Our results agrees well with theoretically calculated values [5]. The floating potential and comparison with theoretical values been tabulated in Table 1 . These results clearly indicate the reduction in plasma sheath potential and its dependence on ion-induced electron emission.

In all cases (for any value of the ion-induced electron emission coefficient), the potential decreases monotonically from the sheath edge to the wall as shown schematically in Fig. 4.

The applied kinetic model is expected to give better insight to the sheath structure. In the present work, we have assumed that the ion-induced electron beams to be cold. This work may be extended to higher dimensional analysis including the effect of oblique magnetic field and the effect of electron and other impurities-induced electrons.

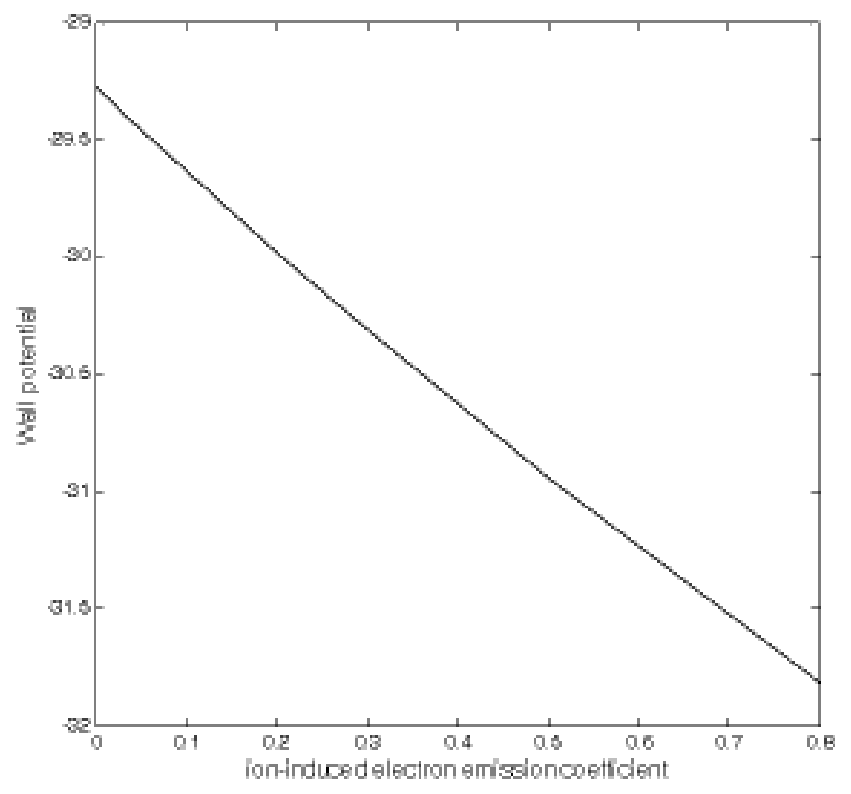

Fig. 3: Wall potential for different valnes of ion-indnced electron emission coefficient 


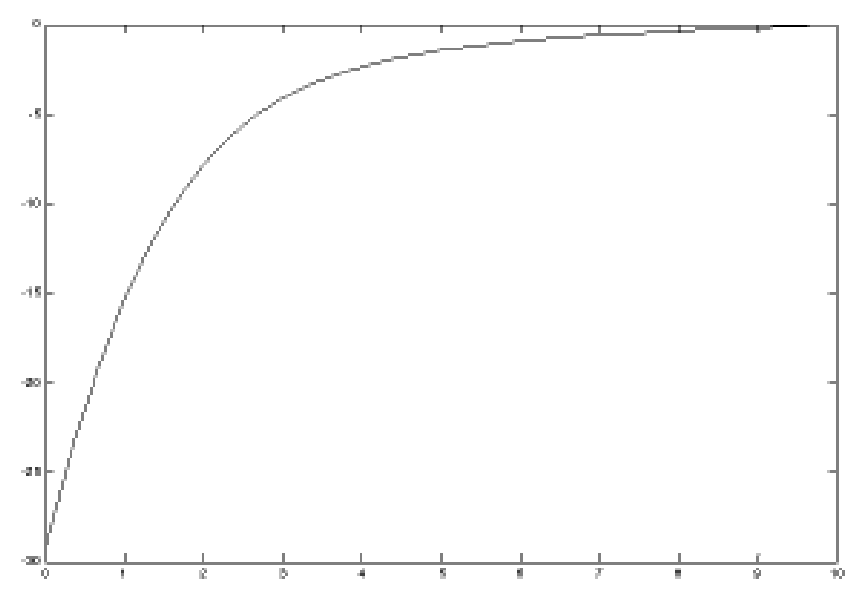

$x$ / Debye length

Fig. 4: Typical self-consistent potential profile in the sheath region

\section{ACKNOWLEDGEMENT}

The support provided by TWAS, the academy of sciences for the developing world, under the TWAS-UNESCO Associateship scheme is highly acknowledged. I would also like to thank the Department of Physics and International Relations Cell of Indian Institute of Science, Bangalore, India for providing the local hospitality.

\section{REFERENCES}

[1] Bohm, D. 1949. The characteristics of electrical diacharges in mageretic fieidz, Edited by A. Guthy and P. K. Wakerling. Mc-Gran Hill, 77.

[2] Rieman, K. U. 1991. J. Plys. D: Appl Phys. 24: 493.

[3] Riemanu, K. U. 2000. J. Tech. Phys. 41 (1): 89.

[4] Nhwal, R. 2006. Scientific World 4 (4): 13

[5] Schupfer, N., Khamal, R et al. 2006. Plasma Phys, Control Fusion 48: 1093

[6] Khawal, R. 2003. A kinetic trajectory simulation madel for bounded plamas, PhD Thesis, University of Imsbruck, Austria

[7] Joshi, R et al 2006. Am. Proc of Nepal Plyysical Society 22 (1) 37.

[8] Bhattarai, S. K. 2006. Effect on the plasma sheath struchure due to the temperature variation of the in-streaning particles, M Sc Dissertation. Tribhuran Univergity, Kirtipu, Kathuandn, Nepal.

[9] Bhattavi, S. K, Joshi, R and Shanal, R. 2007, Scientific World 5 (5) 10 